\title{
Methodologies to generate, extract, purify and fractionate yeast ECM for analytical use in proteomics and glycomics
}

\author{
Fábio Faria-Oliveira' ${ }^{1}$ Joana Carvalho' ${ }^{1}$ Celso LR Belmiro², Montserrat Martinez-Gomariz ${ }^{3}$, Maria Luisa Hernaez ${ }^{3}$,
} Mauro Pavão ${ }^{4}$, Concha Gil ${ }^{3,5}$, Cândida Lucas ${ }^{1}$ and Célia Ferreira ${ }^{1 *}$

\begin{abstract}
Background: In a multicellular organism, the extracellular matrix (ECM) provides a cell-supporting scaffold and helps maintaining the biophysical integrity of tissues and organs. At the same time it plays crucial roles in cellular communication and signalling, with implications in spatial organisation, motility and differentiation. Similarly, the presence of an ECM-like extracellular polymeric substance is known to support and protect bacterial and fungal multicellular aggregates, such as biofilms or colonies. However, the roles and composition of this microbial ECM are still poorly understood.

Results: This work presents a protocol to produce S. cerevisiae and C. albicans ECM in an equally highly reproducible manner. Additionally, methodologies for the extraction and fractionation into protein and glycosidic analytical pure fractions were improved. These were subjected to analytical procedures, respectively SDS-PAGE, 2-DE, MALDI-TOF-MS and LC-MS/MS, and DAE and FPLC. Additional chemical methods were also used to test for uronic acids and sulphation.

Conclusions: The methodologies hereby presented were equally efficiently applied to extract high amounts of ECM material from S. cerevisiae and C. albicans mats, therefore showing their robustness and reproducibility for yECM molecular and structural characterization. yECM from S. cerevisiae and C. albicans displayed a different proteome and glycoside fractions. S. cerevisiae yECM presented two well-defined polysaccharides with different mass/charge, and C. albicans ECM presented a single different one. The chemical methods further suggested the presence of uronic acids, and chemical modification, possibly through sulphate substitution.

All taken, the procedures herein described present the first sensible and concise approach to the molecular and chemical characterisation of the yeast ECM, opening the way to the in-depth study of the microbe multicellular aggregates structure and life-style.
\end{abstract}

\section{Background}

In multicellular organisms, communication between cells is essential, and is profoundly influenced by the extracellular matrix (ECM) components. This scaffolding structure coordinates the biochemical reactions of the different types of cells within the tissues and organs [1]. The mammalian ECM is composed by a wide array of functional molecules, biochemically and biophysically diverse, including proteins, glycosaminoglycans and proteoglycans.

\footnotetext{
*Correspondence: celiamjf@gmail.com

'CBMA (Centre of Molecular and Environmental Biology), Department of Biology, University of Minho, Braga, Portugal

Full list of author information is available at the end of the article
}

The glycosaminoglycans (GAGs) are some of the most dominant ECM components, greatly influencing the cellular behaviour. These highly charged polysaccharides, frequently sulphated, can be found covalently attached to protein cores, forming proteoglycans (PGs), which regulate the GAG distribution and turnover [2]. A high molecular diversity arises from the different combinations of PGs protein cores with one or more types of GAGs chain. These are responsible for the wide variety of biological roles, including structural scaffolding, signalling and growth factor storage [3].

Bacteria and fungi are able to form multicellular threedimensional communities, such as stalks [4,5], mats/biofilms 
$[6,7]$ and colonies [8-10]. These are supported and protected by an extracellular polymeric substance, or ECM, which originates from cellular synthesis and secretion processes, as well as from the lysis of embedded cells. When these microbial multicellular communities developed in natural environments, their ECM components account as well with contributions from the surrounding environment, for example from an infection host [11-13].

The ECM from Saccharomyces cerevisiae colonies revealed the presence of proteins [14], some of which highly glycosylated [15], which remain unidentified. In Candida ECM biofilms, several studies also reported the presence of proteins, as well as polysaccharides and DNA [16-18]. Importantly, an exopolysaccharide composed of $\alpha$-D-glucose and $\beta$-D-glucose, $\alpha$ - $D$-mannose, $\alpha$ - $L$-rhamnose and $\mathrm{N}$-acetyl glucosamine was identified in the ECM from a C. albicans biofilm recovered from an infected intrauterine device [12]. Additionally, the ECM-like substance from flocs of $S$. cerevisise, overexpressing FLO1, were shown to be composed of glucose and branched mannose [19]. Overall, the major players of the yeast colonies and biofilms remain unknown. Therefore, the identification of the molecules composing yeast ECM will contribute for the future understanding of cell-cell communication and other multicellular aggregation processes, namely quorum sensing.

This work provides the foundations for the detailed identification of yeast ECM (yECM) components, presenting the development of a protocol to produce yECM, which robustness was challenged through inter-species reproducibility. Subsequently, methodologies for yECM extraction and fractionation were optimized, providing unprecedented analytical-grade protein and sugar fractions for chromatographic assessment. These methodological advances will open the way to future research on the processes and players of eukaryotic multicellular life-style.

\section{Results and discussion}

\section{Overlay development and ECM extraction}

Molecular and structural assessment of yECM components requires a high amount of cells-free homogenous material, chemically purified into fractions proper for applying analytical techniques. Therefore, a critical step is the reproducible growth of yeast onto large multicellular aggregates without risking large biological heterogeneity between cells, either in very diverse metabolic states and/or different life cycle phases. S. cerevisiae growth onto biofilms is not common. Previous studies showed that the yECM recovered from a S. cerevisiae single colony, or a set of some colonies, was not enough for the in-depth analysis of its components [14]. The development of giant colonies, originated from a drop with several thousand cells, was the most common approach to overcome this obstacle $[7,20]$. We took this concept one step further and inoculated enough cells to produce a whole-plate three-dimensional overlay. The method was tested with wild type strains of $S$. cerevisiae and C. albicans.

Cells collected from a single colony were grown overnight until exponential growth phase, and these fully active cells were used to inoculate homogeneously the whole surface of the YPDa plates. The plates were firstly dried under a sterile air flow until the inoculum was properly absorbed and then incubated at $30^{\circ} \mathrm{C}$. The cell growth and overlay development was followed until the so-called "mature overlay" presented a high quantity of biomass. This happened as a result of 7 days of growth for the case of $S$. cerevisiae, and 5 days growth in the case of $C$. albicans, consistently with the latter being a faster grower. Longitudinal slices of these cultures are shown in Figure 1. The further development of the culture into more days of incubation was assessed, yet resulted in an increased accumulation of dead cells (lysis) in the interior of the aggregate as monitored by flow cytometry (not shown), which is in accordance with previous works [13,21]. Multicellular aggregates in their innate environment, during development and maturation, will inevitably contain intracellular material deriving from cellular debris originated from cell death $[13,18,21]$, which may eventually play some role in the aggregate biological properties. Nevertheless, the method hereby devised aimed at avoiding as far as possible this "contamination" so to assess the basic components of yECM.

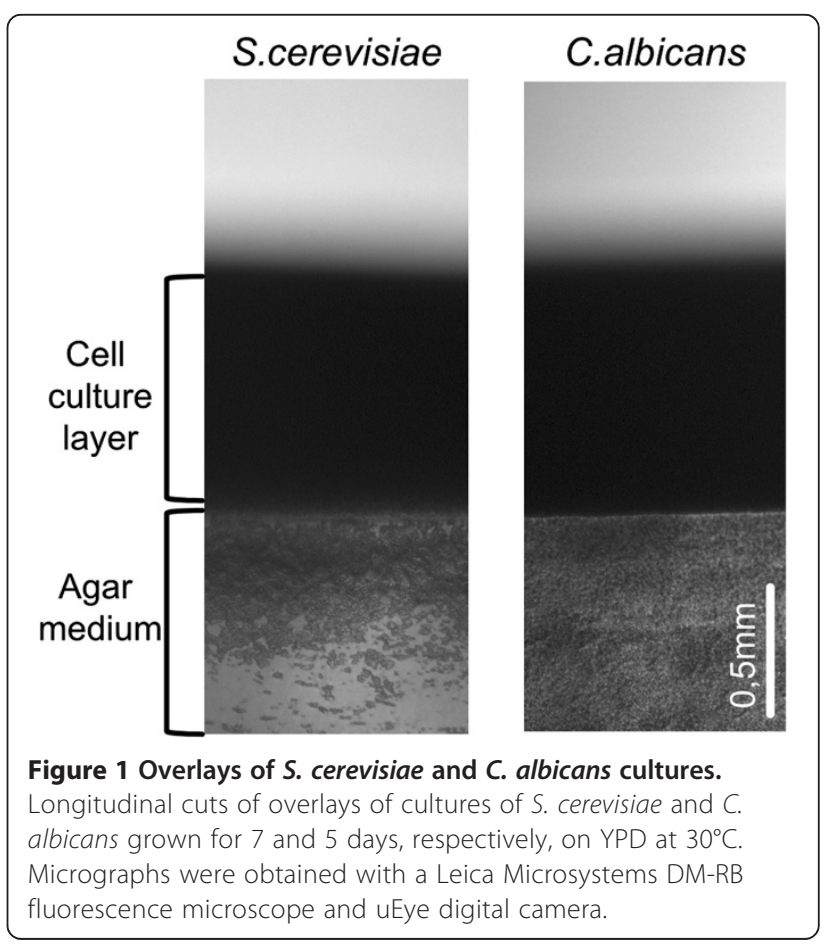


The biomass from the mature overlay was carefully collected through washes with PBS buffer. Considering the interference of $\mathrm{NaCl}$ in several biochemical analysis techniques, namely 2DE and NMR, and in order to maximize the utilisation of yECM samples by a large variety of techniques, the amount of $\mathrm{NaCl}$ in the $\mathrm{PBS}$ buffer was reduced to $100 \mathrm{mM}$. The cellular suspension was very gently placed into a roller incubator to promote the solubilisation of the several molecules present in the intercellular space, followed by centrifugation at $15.000 \mathrm{rpm}$ to induce the deposition of the cells and insoluble molecules. The supernatant was further cleaned by filtration, reducing the possibility of drag intracellular material of the next steps. The filtered supernatant was subsequently lyophilized to enable the concentration of the sample and the resuspension in the appropriate buffers for the following phases of the protocol.

The above-mentioned procedures allowed to retrieve the $S$. cerevisiae and C. albicans overlay biofilm-like yECM in high amounts, crucial for their molecular and structural-characterisation, while ensuring reproducibility between samples, and a low level of putative sample contamination with intracellular contents.

\section{ECM purification and analysis Proteins}

Few studies addressed the proteins present in the extracellular space of microbial multicellular communities, and most of such studies focus on liquid cultures [22-24]. In $S$. cerevisiae colonies, proteins and glycoproteins from the extracellular environment were briefly assessed $[14,20]$. The hereby-developed protocol recovered the proteins present in the $\mathrm{yECM}$ and allowed a proteomics approach.

The samples intended for the precipitation and recovery of proteins were collected in the presence of a cocktail of protease inhibitors (see Methods), precluding the action of proteases, remodelling enzymes or others in yECM. Precipitation was done using the chloroform/ methanol protocol by Wessel and Flugge [25]. This protocol was chosen to avoid the presence of salt and other contaminants detrimental to the protein analysis and identification procedures. A high amount of proteins was recovered, based on the different solubility in diverse solvents. The current protocol avoids the addition of compounds such as TCA (trichloroacetic acid) or DOC (2,5-dimethoxy-4-chloroamphetamine) that compromise the downstream analysis. Furthermore, this protocol in comparison with TCA-based protocols, presents the extra advantage of not promoting the formation of an insoluble pellet, therefore maximizing protein recovery. Importantly, this method also allows the resuspension of the proteins directly in the sample buffer, after evaporation of the solvents.
The isoelectric focusing (IEF)-compatible buffer mentioned in Materials and Methods presents a high protein solubilisation capacity and is compatible with the most common protein quantification methods, namely with Bradford quantification [26]. Moreover, this sample buffer is well suited for both SDS-PAGE and 2DE (Figure 2). This guaranties that the same sample can be analysed by both techniques, without resorting to different buffers that may not solubilize with the same efficiency.

The combination of all these procedures results in a highly reproducible methodology, which allows the analysis of ECM from different yeast strains with similar success (Figure 2A). Importantly, the protein samples obtained through such methodology are pure enough to be analysed by $2 \mathrm{DE}$, known for its sensitivity to detergents, salts, and other molecules that interfere with the proteins' isoelectric point. Besides, those samples can further be subjected to more sensitive techniques as 2DDIGE or mass spectrometry. Figure 2B displays the 2DE gel of $S$. cerevisiae yECM protein sample. The two dimension separation of the proteins also allowed the unambiguous identification of several proteins present in the S. cerevisiae ECM, the glycolytic Tdh3 (glyceraldehyde3-phosphate dehydrogenase), Tpil (triose phosphate isomerase), Pdc1 (pyruvate decarboxylase 1), Eno1/2 (enolase 1 and 2), Sod2 (superoxide dismutase 2), Hsp26 (heatshock protein 26), and Cpr1 (cytoplasmatic peptidyl-prolyl cistrans isomerase) (Figure 2B). Some of these proteins were previously described in the extracellular environment by several authors (reviewed by [27]). Namely, Tdh3 was found overexpressed in the ECM of C. albicans biofilms [28], and enolase has been reported externally located in both yeasts and mammalian cells [23]. In yeasts, proteins found using the present procedures may derive from the outer layers of the cell wall as previously suggested (reviewed by [27]). Their location in vivo has though to be secured by loose attachment, since the present procedures preclude the extraction of covalently linked cell wall materials that require harsh methods to be extracted $[29,30]$. The identification of all the proteins present in yECM, will be possible analysing all the spots present in the $2 \mathrm{DE}$, or using a high throughput technique, as liquid chromatography coupled with tandem mass spectrometry (LC-MS/MS).

\section{Polysaccharides}

Similarly to what occurs with the proteins, little information is available on the polysaccharides of yECM, other than the presence of a few glycoproteins in S. cerevisiae colonies, and the identity of some components of C. albicans biofilms $[12,14,20]$. The sugar fraction of yECM was therefore collected and analysed, for which the procedures widely used to extract the high Eukaryotes ECM were adjusted [31-33]. The samples were 


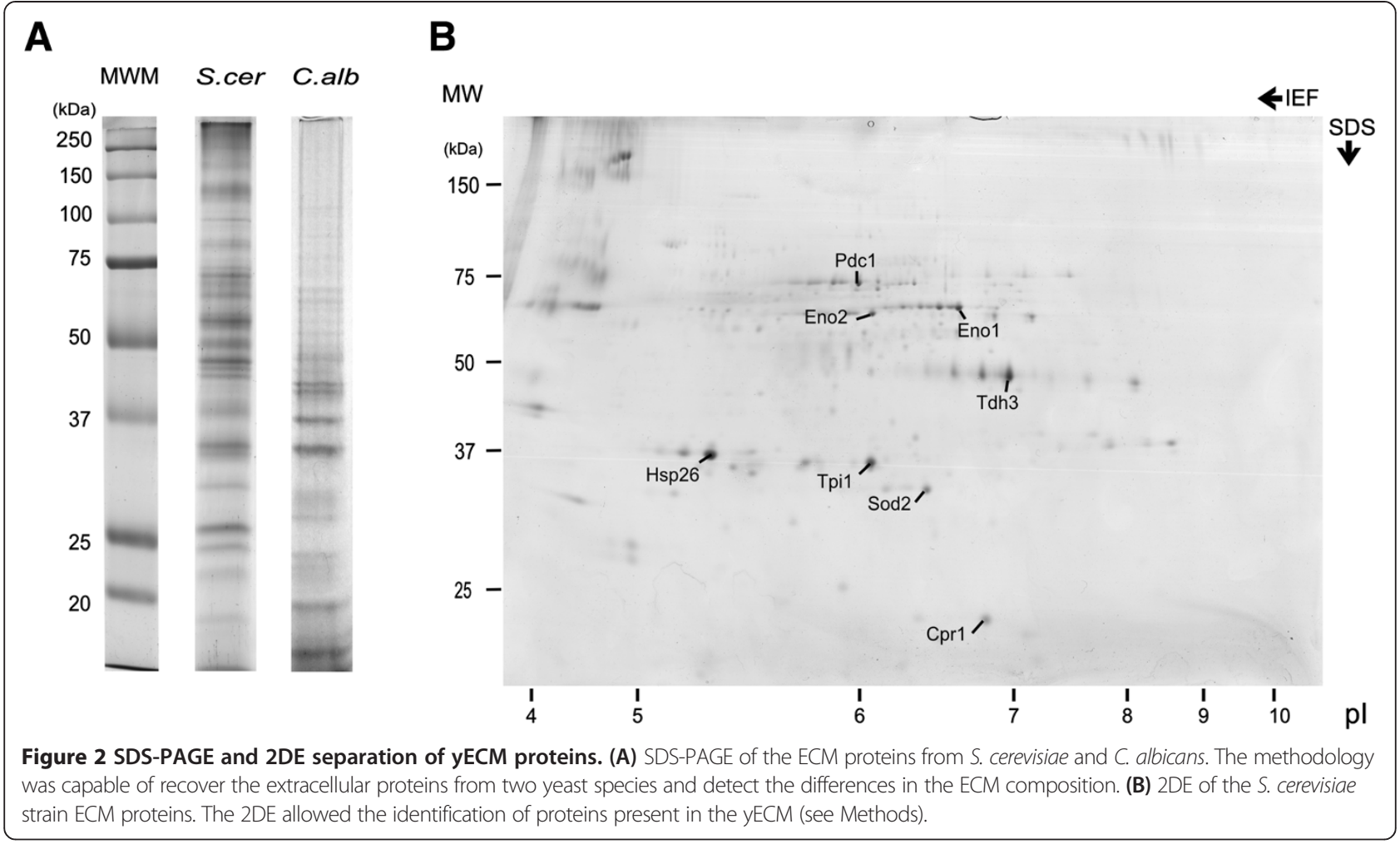

subjected to the action of the broad range protease papain, eliminating the proteins and releasing polysaccharides that might be attached to them. This reaction was performed overnight to warrant complete digestion. After protein separation by centrifugation, the samples containing solubilized polysaccharides were treated with ethanol overnight at $4^{\circ} \mathrm{C}$. Ethanol, disrupts the hydration of the polysaccharide and the charged ions, and when combined with low temperatures, maximizes the precipitation of both neutral and acidic polysaccharides. The utilisation of ethanol in this step is also beneficial because it is easily removable by evaporation. The precipitated polysaccharides can be dissolved, without difficulty, in deionized water or other required solution for the subsequent analysis.

The $S$. cerevisiae and C. albicans yECM polysaccharides recovered by this procedure were evaluated using 1,3diaminopropane acetate agarose electrophoresis (DAE) (Figure 3A). This technique allows the separation of compounds with different degrees of chemical substitution, and is commonly used to separate sulphated polysaccharides [34]. S. cerevisiae presented two differently migrating compounds, whereas using $C$. albicans only one compound was detected. This suggests that the $y E C M$ composition is different from species to species. Importantly, the methodology proved capable of detecting those differences, showing its appropriateness for the study of yECM.
S. cerevisiae ECM polysaccharides were further analysed by fast performance liquid chromatography (FPLC) (Figure 3B). Identically to DAE, this technique also separates polysaccharides through the total charge-to-mass ratio. In this way, fractions of $S$. cerevisiae were collected and tested for (1) total sugars through reaction using sulphuric acid:phenol reaction [35], (2) uronic acids, through carbazole method [36], and (3) possible chemical substitution, analysing the metachromatic shift of 1,9-dimethylmethylene blue (DMMB) [37] (Figure 3B). In total accordance with the DAE results, the $S$. cerevisiae FPLC profile shows two major peaks (dark circles), indicating the existence of at least two different polysaccharide species. The presence of some kind of uronic acid in their composition (white circles), is suggested by carbazole staining, and chemical substitution by the presence of metachromasia (black line). Uronic acids are very common in extracellular polysaccharides, both in microorganisms and higher Eukaryotes [38,39]. Conversely, sulphation is the most frequent chemical substitution of higher Eukaryotes ECM polysaccharides [2].

\section{Conclusions}

The present work provides the foundations for the detailed identification of yECM components and the understanding of its biological functions. A novel and simple protocol to produce yECM from overlays/mats 


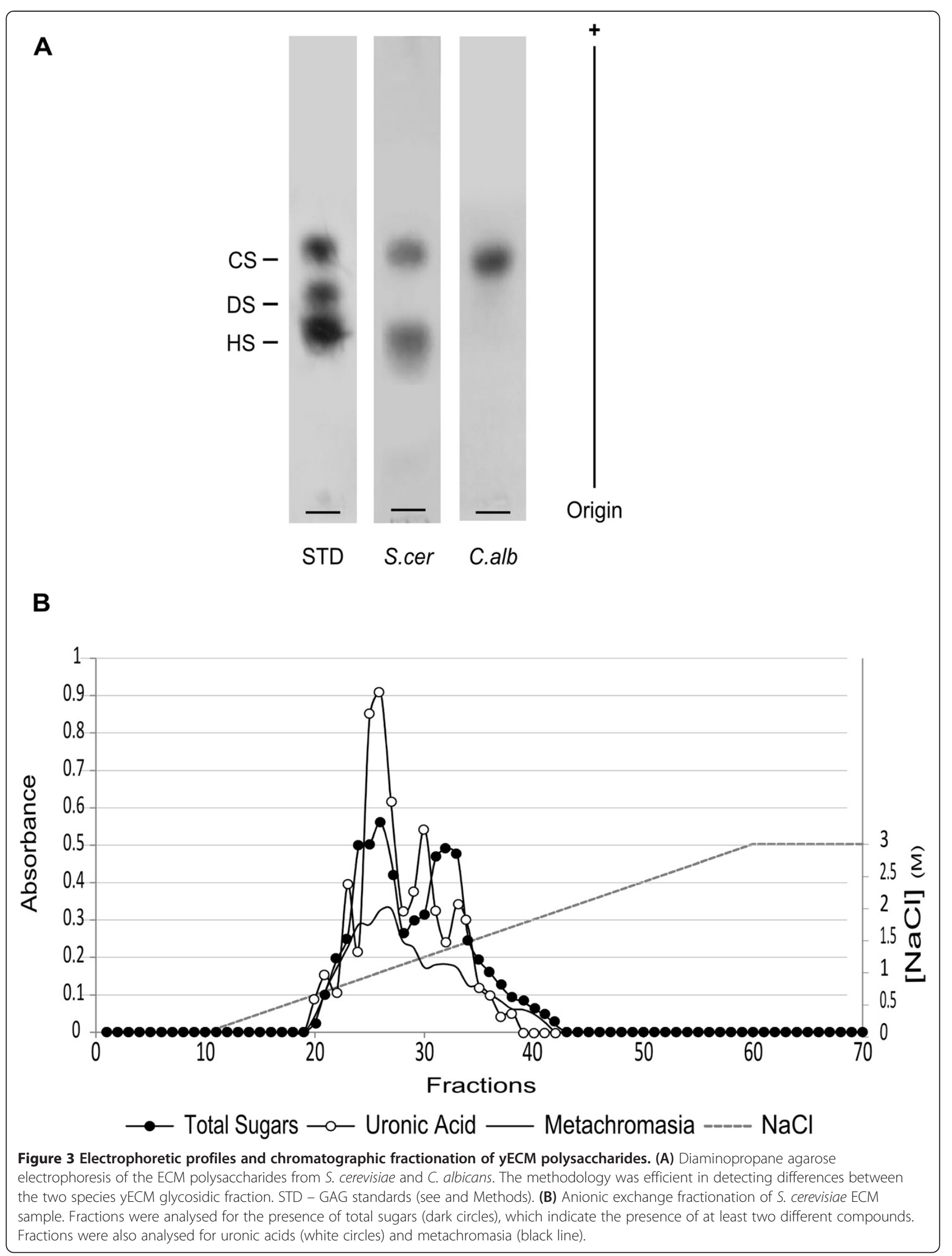


multicellular aggregates was developed. Its robustness was challenged through inter-species reproducibility, using S. cerevisiae and C. albicans. The overlay culture mimicry of a multicellular aggregate warrants the future study of yECM development and regulation, through easy modulation of the microbial culture conditions or yeast genetic background. Moreover, methodologies for yECM extraction and fractionation were optimized, providing analytical-grade protein and sugar fractions, in view of its further assessment by powerful chromatographic analysis, as far as NMR. The procedures in this work consist in the first sensible and easy to use approach to support molecular and chemical characterisation of the yECM, opening the way to the in-depth study of the microbe multicellular aggregates structure and lifestyle.

\section{Methods}

\section{Strains and media}

Yeast strains used in this work were S. cerevisiae W303 (MATa leu2-3 leu2-112 ura3-1 trp1-1 his3-11 his3-15 ade2-1 can1-100) [40] and C. albicans BWP17 (ura3:: imm434/ura3::imm434 iro1/iro1::imm434 his1::hisG/his1:: hisG arg4/arg4) [41]. Cells were grown on rich medium, YPD (yeast extract $1 \%$; peptone $2 \%$; glucose $2 \%$; adenine hemisulphate $0.005 \%$ ) on an orbital shaker, $200 \mathrm{rpm}$, at $30^{\circ} \mathrm{C}$. Growth was monitored by optical density (OD) at $600 \mathrm{~nm}$. Solid growth was performed in YPD supplemented with agarose $(2 \%, \mathrm{w} / \mathrm{v})$ - YPDa. All ingredients percentages are presented as weight per volume units.

\section{Overlay development}

The development of a three-dimensional overlay was preceded by batch cultures grown overnight on liquid YPD, with an air:liquid ratio of 2:1. An inoculum of $1.5 \mathrm{ml}$ batch cultures at $\mathrm{OD}_{600}=1$ was spread in $\mathrm{YPDa}$ plates, using circular movements to ensure an even distribution. The plates were left to completely absorb the inoculum under a laminar flow. The dried plates were incubated at $30^{\circ} \mathrm{C}$ and the overlay was allowed to develop for 7 days (S. cerevisiae) and for 5 days (C. albicans). Longitudinal cuts of the agar and cellular overlay were placed onto a slide and observed under a Leica Microsystems DM-RB fluorescence microscope with a magnifying lens N PLAN 5x/0.11 (Leica P/N 506029), in bright field. Images were acquired through an IDS uEye USB 2.0 camera and processed with uEye Cockpit Application Software.

\section{Matrix extraction}

The cellular overlay was carefully collected into a $50 \mathrm{ml}$ Falcon tube with PBS buffer ( $\mathrm{NaCl} 100 \mathrm{mM} ; \mathrm{KCl} 2.7 \mathrm{mM}$; $\mathrm{Na}_{2} \mathrm{HPO}_{4} \cdot 2 \mathrm{H}_{2} \mathrm{O} 10 \mathrm{mM} ; \mathrm{KH}_{2} \mathrm{PO}_{4} 2.0 \mathrm{mM}$; pH 7.4). The collected biomass was incubated for 10 minutes with constant rotation in a tube roller (SRT1; Stuart, Staffordshire,
UK) to allow the complete suspension of proteins and polysaccharides. The samples intended for protein purification and analysis, were supplemented with a protease inhibitor cocktail (PMSF $0.2 \mu \mathrm{g} / \mathrm{ml}$; Aprotinin $0.32 \mu \mathrm{g} / \mathrm{ml}$; Pepstatin $1 \mu \mathrm{g} / \mathrm{ml}$; Leupeptin $1 \mu \mathrm{g} / \mathrm{ml}$ ). The suspension, containing cells and extracellular matrix, was then centrifuged for 10 minutes at $15,000 \mathrm{rpm}$ and $4^{\circ} \mathrm{C}$ in a Sigma 4-16 K centrifuge (Sigma, Osterode, Germany) to promote the deposition of the insoluble material. The supernatant was collected, filtered through a $0.45 \mu \mathrm{m}$ membrane and freeze-dried (Christ Alpha 2-4 Christ LDC-1 m, B. Braun). Membrane integrity was assessed by cytometry as described before [42]. Briefly, cells were harvested and added $4 \mu \mathrm{g} / \mathrm{ml}$ propidium iodide (Sigma). After 10 min incubation in the dark at room temperature, the samples were analysed in an Epics ${ }^{\circ} \mathrm{XL}^{\mathrm{mm}}$ (Beckman Coulter) flow cytometer.

\section{ECM precipitation and recovery}

Proteins - The lyophilized extract was resuspended in MilliQ water (just enough volume to completely solubilize the overlay components). The proteins were precipitated using the chloroform/methanol protocol [25]. The resulting pellet was left at room temperature to evaporate the remaining methanol, and then resuspended in isoelectric focusing IEF buffer (urea $7 \mathrm{M}$; thiourea $2 \mathrm{M}$; CHAPS $2 \%, \mathrm{w} / \mathrm{v}$ ) [43], which is compatible with downstream analysis. Alternatively, Laemmli buffer (Tris- $\mathrm{HCl} 125 \mathrm{mM}$, pH 6.8; glycerol 20\%, w/v; SDS 4\%, w/v), modified through the further addition of $6 \mathrm{M}$ urea and 1\% w/v DTT- ML buffer, was used with similar efficiency for SDS-PAGE purposes. Protein was quantified with Bio-Rad Protein Assay (Bio-Rad, Richmond, CA, USA) as recommended by the manufacturer.

Polysaccharides - The lyophilized supernatant was resuspended in digesting buffer (NaOAc 100 mM; EDTA $5 \mathrm{mM}$; cysteine $5 \mathrm{mM}$; pH 5.5) in a proportion of $20 \mathrm{ml}$ per gram of dry weight. Double-crystalized papain (Merck, Darmstadt) was added to the mixture $(10 \mathrm{mg} / \mathrm{ml})$ and incubated at $60^{\circ} \mathrm{C}$ overnight. The mixture was centrifuged (3,000 rpm for 10 minutes at room temperature) and the clear supernatant was collected. The recovery of yECM polysaccharides was achieved through ethanol precipitation. Three volumes of ethanol $(95-99 \%, v / v)$ were added to the supernatant and incubated overnight at $4^{\circ} \mathrm{C}$. The precipitate was collected by centrifugation, 10 minutes at $3,000 \mathrm{rpm}$, and left at room temperature until total evaporation of the residual ethanol. An aliquot of the pellet was resuspended in deionised water and was evaluated for (i) total sugar content, by the reaction of phenol-sulphuric acid with neutral sugars [25], (ii) the presence of hexuronic acids, through carbazole method [26], and (iii) chemical substitution by metachromatic shift of 1,9dimethylmethylene blue (DMMB) [27]. 


\section{ECM analysis \\ Proteins}

One and two dimensions electrophoresis - The analysis of the protein profiles was performed by one-dimensional electrophoretic separation under denaturing conditions (SDSPAGE) in a Mini PROTEAN ${ }^{\circ} 3$ Cell apparatus (Bio-Rad) and by 2DE in a DALTSix system (GE Healthcare).

The SDS-PAGE was carried out in a $1.5 \mathrm{~mm}$ thick polyacrylamide gel, with a $4 \%$ stacking gel and a $10 \%$ resolving gel. The sample $(5 \mu \mathrm{g})$ was brought up to $10 \mu \mathrm{l}$ with MilliQ water and mixed with loading buffer, Laemmli $5 \mathrm{X}$ [44]. The mixture was boiled for 5 minutes and then transferred to ice, for at least 5 minutes. The sample was loaded and the electrophoresis was run at $100 \mathrm{~V}$, for 2 hours or until the migration front reached the end of the gel.

For the 2DE, the samples were primarily submitted to IEF - first dimension - and then run according to molecular weight in a homogeneous polyacrylamide gel (10\% of total polyacrylamide and $2.6 \%$ of bis-acrylamide $10 \% \mathrm{~T}, 2.6 \% \mathrm{C}$ ) - second dimension. The IEF dry strips (24 cm, pH 3-11NL) were incubated in Rehydration buffer (urea $8 \mathrm{M}$; thiourea $2 \mathrm{M}$; CHAPS 4\%, w/v; IPGphor buffer pH 3-11 2\%, w/v; DeStreak 1.5\%, w/v) for at least 8 hours. The sample, mixed with a 2X IPGphor buffer pH 3-11/ DTT solution, was applied by cup loading. The IEF was performed at $20^{\circ} \mathrm{C}$ in an Ettan IPGphor 3 apparatus (GE Healthcare), according to the following program: $120 \mathrm{~V}$ for $1 \mathrm{~h}$; $500 \mathrm{~V}$ for $2 \mathrm{~h}$; $500-1000 \mathrm{~V}$ in gradient for $2 \mathrm{~h}$; $1000-5000 \mathrm{~V}$ in gradient for $6 \mathrm{~h} ; 5000 \mathrm{~V}$ for $10 \mathrm{~h}$. After this, strips were equilibrated first in reducing solution (urea $6 \mathrm{M}$; Tris- $\mathrm{HCl}$ pH $6.850 \mathrm{mM}$; glycerol 30\%. v/v; SDS $2 \%, \mathrm{w} / \mathrm{v}$; dithiothreitol $2 \%, \mathrm{w} / \mathrm{v}$ ) for 12 minutes, and then 5 minutes in alkylating solution (Urea $6 \mathrm{M}$; Tris-HCl pH 6.850 mM; glycerol 30\%; v/v; SDS 2\%, w/v; iodoacetamide $2.5 \%, \mathrm{w} / \mathrm{v})$. Second dimension SDS-PAGE were run on homogeneous polyacrylamide gel $(12 \% \mathrm{~T}$, $2.6 \% \mathrm{C}$ ). Electrophoresis was carried out at $18^{\circ} \mathrm{C}, 1 \mathrm{~W} /$ gel for 18 hours, using Ettan DALTsix system (GE Healthcare). Both SDS-PAGE and 2DE gels were stained according to the Colloidal Coomassie Blue protocol $[45,46]$, and scanned with a calibrated densitometer (Bio-Rad; Molecular Imager GS-800).

Protein Identification - The chosen spots from the 2DE gels were excised and in-gel digested [47]. Samples were reduced with $10 \mathrm{mM}$ DTT, in ammonium bicarbonate (25 mM; pH 8.5), for 30 minutes at $56^{\circ} \mathrm{C}$ and subsequently alkylated with $55 \mathrm{mM}$ iodoacetamide, in ammonium bicarbonate ( $25 \mathrm{mM}$; pH 8.5), for 20 minutes in the dark. Finally, samples were digested with $12.5 \mathrm{ng} / \mu \mathrm{l}$ of sequencing grade trypsin (Roche Molecular Biochemicals), in ammonium bicarbonate ( $25 \mathrm{mM} ; \mathrm{pH} 8.5)$, overnight at $37^{\circ} \mathrm{C}$.

The spots supernatants were collected and $1 \mu$ was spotted onto a MALDI target plate and allowed to air- dry at room temperature. To each digested spot was added $0.5 \mu \mathrm{l}$ of $\alpha$-cyano-4-hydroxytranscinnamic acid matrix (Sigma; $3 \mathrm{mg} / \mathrm{ml}$ in acetonitrile 50\%; v/v), and allowed again to air-dry at room temperature. MALDITOF MS analyses were performed in a MALDI-TOF/ TOF mass spectrometer 4800 plus Proteomics Analyzer (Applied Biosystems. MDS Sciex, Toronto, Canada) and 4000 Series Explorer ${ }^{\mathrm{TM}} \mathrm{v}$ 3.5 Software (ABSciex). The instrument was operated in reflector mode, with an accelerating voltage of $20000 \mathrm{~V}$. All mass spectra were internally calibrated using peptides from the autodigestion of the trypsin. MALDI-TOF MS analysis produces peptide mass fingerprints, which can be collected and represented as a list of monoisotopic molecular weights with a Signal to Noise ratio greater than 12 . The suitable precursors for MS/MS sequencing analysis were selected and fragmentation was carried out using the Collisioninduced dissociation on (atmospheric gas) $1 \mathrm{kV}$ ion reflector mode and precursor mass Windows $+/-4$ Da. The plate model \& default calibration were optimized for the MS-MS spectra processing. The search of peptides was performed in batch mode using GPS Explorer v3.5 software (ABSciex) with a licensed version 2.3 of MASCOT (www.matrixscience.com), using the NCBInr database (date: 08052012; 17919084 sequences; 6150218869 residues). The MASCOT search parameters were: (1) species: S. cerevisiae; (2) allowed number of missed cleavages: 1 ; (3) fixed modification: carbamidomethyl cysteine, (4) variable modifications: methionine oxidation; (5) peptide tolerance: $\pm 50 \mathrm{ppm}$ for PMF and $80 \mathrm{ppm}$ for MS/MS searches; (6) MS/MS tolerance: $\pm 0.3 \mathrm{Da}$ and (7): peptide charge: +1 . In all identified proteins, the probability score was greater than the one fixed by Mascot as being significant, that is, a $\mathrm{p}$ value under 0.05 .

\section{Polysaccharides}

Electrophoretic analysis - ECM total polysaccharide extracts were submitted to agarose gel electrophoresis as described previously [48]. Briefly, about $1.5 \mu \mathrm{g}$ of the sample (as of uronic acid content) was applied to a $0.5 \%$ agarose gel in a 1,3-diaminopropane acetate buffer $(50 \mathrm{mM}$; $\mathrm{pH}$ 9.0), and run for $1 \mathrm{~h}$ at $100 \mathrm{~V}$. As standard, a mixture of sulphated polysaccharides containing chondroitin sulphate, dermatan sulphate, and heparan sulphate $(1.5 \mu \mathrm{g}$ of uronic acid of each), was used. The polysaccharides were fixed with aqueous $0.1 \%$ cetyl-trimethylammonium bromide solution $\left(\right.$ Cetavlon $\left.^{\circ}\right)$, the gel was allowed to dry, and stained with $0.1 \%$ toluidine blue in acetic acid/ethanol/water $(0.1: 5: 5, \mathrm{v} / \mathrm{v} / \mathrm{v})$.

FPLC - An aliquot of the ethanol-precipitated pellet $(\approx 20 \mathrm{mg})$ was resuspended in $1 \mathrm{ml}$ MilliQ water, filtered through a syringe filter $(0.22 \mu \mathrm{m})$ for analysis in a FPLC system (Pharmacia Biotech, Sweden). The filtered sample was applied to a Hitrap Q-XL column, equilibrated 
with elution buffer (Tris-HCl $20 \mathrm{mM}$; pH 8.6). The polysaccharides were eluted by a linear gradient of $0-3.0 \mathrm{M}$ $\mathrm{NaCl}$, at a flow rate of $0.50 \mathrm{ml} / \mathrm{min}$, and fractions of $0.5 \mathrm{ml}$ were collected. The total sugar content of the fractions was evaluated by the reaction of phenol-sulphuric acid with hexoses [35]. The presence of hexuronic acids was assessed through the carbazole method [36], and the possible chemical substitution, tested by the metachromatic shift of 1,9-dimethylmethylene blue (DMMB) [37].

\section{Abbreviations}

ECM: Extracellular matrix; SDS-PAGE: Sodium dodecyl sulphate polyacrylamide gel electrophoresis; 2-DE: Two-dimensional gel electrophoresis; MALDI-TOF-MS: Matrix-assisted laser desorption-ionization time-of-flight mass spectrometry; LC-MS/MS: Liquid chromatography coupled with tandem mass spectrometry; DAE: 1,3-Diaminopropane acetate agarose electrophoresis; FPLC: Fast performance liquid chromatography;

GAGs: Glycosaminoglycans; PGs: Proteoglycans; yECM: Yeast extracellular matrix; NMR: Nuclear magnetic resonance; IEF: isoelectric focusing; 2 DE-DIGE: Two-dimensional difference gel electrophoresis;

DMMB: dimethylmethylene blue.

\section{Competing interests}

The authors declare that they have no competing interests.

\section{Authors' contributions}

FF-O, JC, CB and CF carried out the experimental studies, having FF-O contributed $70 \%$. CF, MP, CG and CL supervised FF-O, JC, CB, MMG and $\mathrm{MLH}$ and checked the data. FF-O and CF wrote this manuscript. CL and $\mathrm{CG}$ revised the manuscript. All authors read and approved the final manuscript.

\section{Acknowledgements}

Authors would like to acknowledge Joana Tulha for assistance on yeasts overlay photographs, and to Rui Armada for C. albicans ECM SDS-PAGE experiment. The proteomic analysis was carried out at the proteomics facility UCM-PCM, a member of the ProteoRed network. The polysaccharide analysis was performed at the Laboratory of Glycoconjugates Biochemistry and Cellular Biology, UFRJ, Brazil. Fábio Faria-Oliveira was supported by a PhD scholarship SFRH/BD/45368/2008 from FCT (Fundação para a Ciência e a Tecnologia). This work was funded by Marie Curie Initial Training Network GLYCOPHARM (PITN-GA-2012-317297), and by FCT/MEC through Portuguese funds (PIDDAC) PEst-OE/BIA/UI4050/2014. The authors would also like to acknowledge Hugh S. Johnson for critical reading of the manuscript regarding English usage.

\section{Author details}

${ }^{1}$ CBMA (Centre of Molecular and Environmental Biology), Department of Biology, University of Minho, Braga, Portugal. ' Institute of Medical Biochemistry, Laboratory of Glycoconjugates Biochemistry and Cellular Biology, Federal University of Rio de Janeiro/ Polo de Macaé, Macaé, Brazil. ${ }^{3}$ Unidad de Proteómica, Universidad Complutense de Madrid - Parque Científico de Madrid UCM-PCM), Madrid, Spain. ${ }^{4}$ Institute of Medical Biochemistry, Laboratory of Glycoconjugates Biochemistry and Cellular Biology, Federal University of Rio de Janeiro, Rio de Janeiro, Brazil. ${ }^{5}$ Departamento de Microbiología II, Facultad de Farmacia, Universidad Complutense de Madrid, Madrid, Spain

Received: 20 May 2014 Accepted: 9 September 2014

Published online: 25 October 2014

\section{References}

1. Nelson DL, Cox MM: Lehninger Principles of Biochemistry. New York: W. H. Freeman; 2008.

2. Esko JD, Kimata K, Lindahl U: Proteoglycans and sulfated glycosaminoglycans. In Essentials of Glycobiology. 2nd edition. Edited by Varki A, Cummings RD, Esko JD, Freeze HH, Stanley P, Bertozzi CR, Hart GW, Etzler ME. New York: Cold Spring Harbor Laboratory Press; 2009:229-248.

3. Schaefer L, Schaefer RM: Proteoglycans: from structural compounds to signaling molecules. Cell Tissue Res 2010, 339(1):237-246.
4. Engelberg D, Mimran A, Martinetto H, Otto J, Simchen G, Karin M, Fink GR: Multicellular stalk-like structures in Saccharomyces cerevisiae. J Bacteriol 1998, 180(15):3992-3996.

5. Scherz R, Shinder V, Engelberg D: Anatomical analysis of Saccharomyces cerevisiae stalk-like structures reveals spatial organization and cell specialization. J Bacteriol 2001, 183(18):5402-5413.

6. Reynolds TB, Fink GR: Bakers' yeast, a model for fungal biofilm formation. Science 2001, 291(5505):878-881.

7. Reynolds TB, Jansen A, Peng X, Fink GR: Mat formation in Saccharomyces cerevisiae requires nutrient and $\mathrm{pH}$ gradients. Eukaryot Cell 2008 , 7(1):122-130

8. Čáp M, Váchová L, Palková Z: How to survive within a yeast colony?: change metabolism or cope with stress? Commun Integr Biol 2010, 3(2):198-200.

9. Palková Z, Váchová L: Life within a community: benefit to yeast long-term survival. FEMS Microbiol Rev 2006, 30(5):806-824

10. Vachová L, Čáp M, Palková Z: Yeast colonies: a model for studies of aging, environmental adaptation, and longevity. Oxid Med Cell Longev 2012, 2012(1):601836.

11. Sutherland I: Biofilm exopolysaccharides: a strong and sticky framework. Microbiology 2001, 147(Pt 1):3-9.

12. Lal $P$, Sharma D, Pruthi $P$, Pruthi V: Exopolysaccharide analysis of biofilm-forming Candida albicans. J Appl Microbiol 2010, 109(1):128-136.

13. Nobile CJ, Mitchell AP: Microbial biofilms: e pluribus unum. Curr Biol 2007 , 17(10):R349-R353.

14. Kuthan M, Devaux F, Janderova B, Slaninova I, Jacq C, Palková Z Domestication of wild Saccharomyces cerevisiae is accompanied by changes in gene expression and colony morphology. Mol Microbio/ 2003, 47(3):745-754.

15. Váchová L, Štovíček V, Hlaváček O, Chernyavskiy O, Štěpánek L, Kubínová L, Palková Z: Flo11p, drug efflux pumps, and the extracellular matrix cooperate to form biofilm yeast colonies. J Cell Biol 2011, 194(5):679-687.

16. Chandra J, Kuhn DM, Mukherjee PK, Hoyer LL, McCormick T, Ghannoum MA: Biofilm formation by the fungal pathogen Candida albicans: development, architecture, and drug resistance. J Bacteriol 2001, 183(18):5385-5394.

17. Al-Fattani MA, Douglas L: Biofilm matrix of Candida albicans and Candida tropicalis: chemical composition and role in drug resistance. $J$ Med Microbiol 2006, 55(8):999-1008.

18. Martins M, Uppuluri P, Thomas DP, Cleary IA, Henriques M, Lopez-Ribot JL, Oliveira R: Presence of extracellular DNA in the Candida albicans biofilm matrix and its contribution to biofilms. Mycopathologia 2010, 169(5):323-331.

19. Beauvais A, Loussert C, Prevost MC, Verstrepen K, Latge JP: Characterization of a biofilm-like extracellular matrix in FLO1-expressing Saccharomyces cerevisiae cells. FEMS Yeast Res 2009, 9(3):411-419.

20. Stovíček V, Váchová L, Kuthan M, Palková Z: General factors important for the formation of structured biofilm-like yeast colonies. Fungal Genet Biol 2010, 47(12):1012-1022.

21. Cáp M, Vachová L, Palková Z: Yeast colony survival depends on metabolic adaptation and cell differentiation rather than on stress defense. J Biol Chem 2009, 284(47):32572-32581

22. Insenser MR, Hernaez ML, Nombela C, Molina M, Molero G, Gil C: Gel and gel-free proteomics to identify Saccharomyces cerevisiae cell surface proteins. J Proteomics 2010, 73(6):1183-1195.

23. Lopez-Villar E, Monteoliva L, Larsen MR, Sachon E, Shabaz M, Pardo M, Pla J, Gil C, Roepstorff P, Nombela C: Genetic and proteomic evidences support the localization of yeast enolase in the cell surface. Proteomics 2006, 6(Suppl 1):S107-S118.

24. Pardo M, Monteoliva L, Pla J, Sanchez M, Gil C, Nombela C: Two-dimensional analysis of proteins secreted by Saccharomyces cerevisiae regenerating protoplasts: a novel approach to study the cell wall. Yeast 1999, 15(6):459-472.

25. Wessel D, Flugge UI: A method for the quantitative recovery of protein in dilute solution in the presence of detergents and lipids. Anal Biochem 1984, 138(1):141-143.

26. Bradford MM: A rapid and sensitive method for the quantitation of microgram quantities of protein utilizing the principle of protein-dye binding. Anal Biochem 1976, 72(1):248-254.

27. Nombela C, Gil C, Chaffin WL: Non-conventional protein secretion in yeast. Trends Microbiol 2006, 14(1):15-21.

28. Lattif AA, Chandra J, Chang J, Liu S, Zhou G, Chance MR, Ghannoum MA, Mukherjee PK: Proteomics and pathway mapping analyses reveal phase-dependent over-expression of proteins associated with 
carbohydrate metabolic pathways in Candida albicans biofilms. Open Proteomic J 2008, 1(1):5-26.

29. Ferreira C, Lucas C: The yeast $O$-acyltransferase Gup1p interferes in lipid metabolism with direct consequences on the sphingolipid-sterol-ordered domains integrity/assembly. Biochim Biophys Acta 2008, 1778(11):2648-2653.

30. Ferreira C, Silva S, van Voorst F, Aguiar C, Kielland-Brandt MC, Brandt A, Lucas C Absence of Gup 1p in Saccharomyces cerevisiae results in defective cell wall composition, assembly, stability and morphology. FEMS Yeast Res 2006, 6(7):1027-1038.

31. Gomes AM, Kozlowski EO, Pomin VH, de Barros CM, Zaganeli JL, Pavao MS: Unique extracellular matrix heparan sulfate from the bivalve Nodipecten nodosus (Linnaeus, 1758) safely inhibits arterial thrombosis after photochemically induced endothelial lesion. J Biol Chem 2010, 285(10):7312-7323.

32. Belmiro CL, Castelo-Branco MT, Melim LM, Schanaider A, Elia C, Madi K Pavao MS, de Souza HS: Unfractionated heparin and new heparin analogues from ascidians (chordate-tunicate) ameliorate colitis in rats. J Biol Chem 2009, 284(17):11267-11278

33. Pavão MSG, Mourão PAS, Mulloy B, Tollefsen DM: A unique dermatan sulfate-like glycosaminoglycan from ascidian. J Biol Chem 1995, 270(52):31027-31036.

34. Cohen DM, Mourão PAS, Dietrich CP: Differentiation of mucopolysaccharidoses by analyses of the excreted sulfated mucopolysaccharides. Clin Chim Acta 1977, 80(3):555-562.

35. Masuko T, Minami A, Iwasaki N, Majima T, Nishimura S, Lee YC: Carbohydrate analysis by a phenol-sulfuric acid method in microplate format. Anal Biochem 2005, 339(1):69-72.

36. Bitter T, Muir HM: A modified uronic acid carbazole reaction. Anal Biochem 1962, 4(4):330-334

37. Farndale RW, Buttle DJ, Barrett AJ: Improved quantitation and discrimination of sulphated glycosaminoglycans by use of dimethylmethylene blue. Biochim Biophys Acta 1986, 883(2):173-177.

38. Afratis N, Gialeli C, Nikitovic D, Tsegenidis T, Karousou E, Theocharis AD, Pavao MS, Tzanakakis GN, Karamanos NK: Glycosaminoglycans: key players in cancer cell biology and treatment. FEBS J 2012, 279(7):1177-1197.

39. Tielen P, Strathmann M, Jaeger KE, Flemming HC, Wingender J: Alginate acetylation influences initial surface colonization by mucoid Pseudomonas aeruginosa. Microbiol Res 2005, 160(2):165-176.

40. Thomas BJ, Rothstein R: Elevated recombination rates in transcriptionally active DNA. Cell 1989, 56(4):619-630.

41. Wilson RB, Davis D, Mitchell AP: Rapid hypothesis testing with Candida albicans through gene disruption with short homology regions. $J$ Bacteriol 1999, 181(6):1868-1874.

42. Tulha J, Faria-Oliveira F, Lucas C, Ferreira C: Programmed cell death in Saccharomyces cerevisiae is hampered by the deletion of GUP1 gene. BMC Microbiol 2012, 12(1):80.

43. Stanley BA, Eyk JE: A Solubility Optimization Protocol for Two-Dimensional Gel Electrophoresis of Cardiac Tissue. In Cardiovascular Proteomics: Methods and Protocols. Edited by Vivanco F. New Jersey: Humana Press; 2007:59-65.

44. Laemmli UK: Cleavage of structural proteins during the assembly of the head of bacteriophage T4. Nature 1970, 227(5259):680-685.

45. Neuhoff $\mathrm{V}$, Stamm R, Eibl H: Clear background and highly sensitive protein staining with Coomassie Blue dyes in polyacrylamide gels: a systematic analysis. Electrophoresis 1985, 6(9):427-448.

46. Pink M, Verma N, Rettenmeier AW, Schmitz-Spanke S: CBB staining protocol with higher sensitivity and mass spectrometric compatibility. Electrophoresis 2010, 31(4):593-598

47. Havlis J, Thomas H, Sebela M, Shevchenko A: Fast-response proteomics by accelerated in-gel digestion of proteins. Anal Chem 2003, 75(6):1300-1306.

48. Pavão MSG, Aiello KRM, Werneck CC, Silva LCF, Valente A-P, Mulloy B, Colwell NS, Tollefsen DM, Mourão PAS: Highly sulfated dermatan sulfates from ascidians. J Biol Chem 1998, 273(43):27848-27857.

doi:10.1186/s12866-014-0244-0

Cite this article as: Faria-Oliveira et al: Methodologies to generate,

extract, purify and fractionate yeast ECM for analytical use in proteomics and glycomics. BMC Microbiology 2014 14:244.

\section{Submit your next manuscript to BioMed Central and take full advantage of:}

- Convenient online submission

- Thorough peer review

- No space constraints or color figure charges

- Immediate publication on acceptance

- Inclusion in PubMed, CAS, Scopus and Google Scholar

- Research which is freely available for redistribution

Submit your manuscript at www.biomedcentral.com/submit
C Biomed Central 